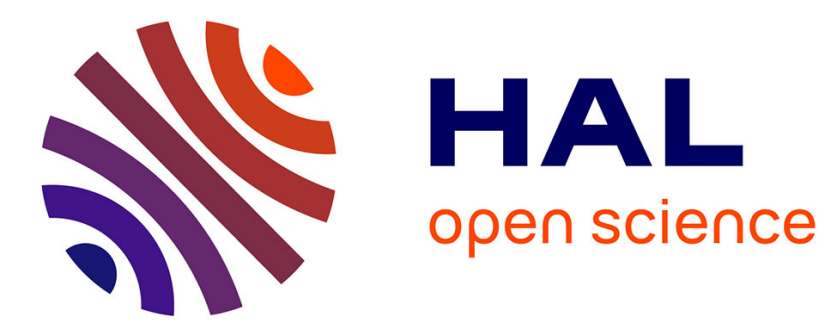

\title{
Algebraicity and the tensor product of concept lattices Bogdan Chornomaz
}

\section{To cite this version:}

Bogdan Chornomaz. Algebraicity and the tensor product of concept lattices. 2014. hal-00983199v1

\section{HAL Id: hal-00983199 \\ https://hal.science/hal-00983199v1}

Preprint submitted on 24 Apr 2014 (v1), last revised 24 May 2014 (v2)

HAL is a multi-disciplinary open access archive for the deposit and dissemination of scientific research documents, whether they are published or not. The documents may come from teaching and research institutions in France or abroad, or from public or private research centers.
L'archive ouverte pluridisciplinaire HAL, est destinée au dépôt et à la diffusion de documents scientifiques de niveau recherche, publiés ou non, émanant des établissements d'enseignement et de recherche français ou étrangers, des laboratoires publics ou privés. 


\title{
ALGEBRAICITY AND THE TENSOR PRODUCT OF CONCEPT LATTICES
}

\author{
BOGDAN CHORNOMAZ
}

\begin{abstract}
In this paper we prove that the tensor product of complete lattices, as it is defined in formal context analysis, preserves algebraicity. The proof of this fact is based on the compactness of propositional logic. We use this property to show that the box product of $(0, \vee)$-semilattices, introduced by G.Grätzer and F.Wehrung in 1999, can be obtained from the tensor product of concept lattices in a manner similar to how it is done in the definition of tensor product in "general" lattice theory.
\end{abstract}

\section{InTRODUCTION}

Traditionally, the tensor product in lattice theory is defined on $(0, \vee)$-semilattices as a join-semilattice of compact bi-ideals in the direct product of the corresponding lattices, see [4]. The formal context analysis provides a different (and nonequivalent) approach toward the concept of tensor product. Namely, the tensor product of context lattices is defined as the concept lattice of the direct product of their formal contexts. Theorem 14 of [3] proves that the resulting lattice is independent of the choice of formal contexts, thus justifying the definition. However, the concept lattices are exactly the complete lattices, and so thus defined tensor product has a narrower scope than that from [4].

In Section 2 we argue that we can define a complete tensor product of complete lattices, denoted $\stackrel{\mathrm{bi}}{\otimes}$, as a set of complete bi-ideals in their direct product, in much the same way as it is done for the tensor product from [4], which we call finite tensor

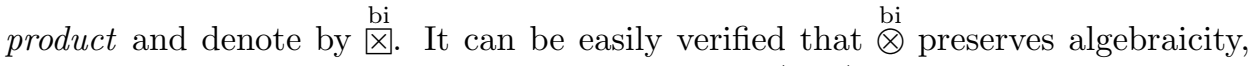
thus enabling the following construction: for any $(0, \vee)$-semilattices $A$ and $B$ we take the complete tensor product of complete algebraic lattices $\operatorname{Id} A$ and $\operatorname{Id} B$, and then take the $(0, \vee)$-semilattice of compact elements of $\operatorname{Id} A \stackrel{\text { bi }}{\otimes} \operatorname{Id} B$. Unsurprisingly, we get

$$
A \bowtie B=\mathrm{bi}(\operatorname{Id} A \stackrel{\text { bi }}{\otimes} \operatorname{Id} B) .
$$

Further on, we will omit the word "complete" whenever the context is clear.

The results of Section 2 are rather trivial and mainly given without the proof. As suggested by the section title, they serve as a motivation for introducing similar construction for the tensor product defined via formal contexts, which we call complete fc-tensor product and denote $\stackrel{\mathrm{fc}}{\otimes}$. The key property of this construction is the preservation of algebraicity, which is trivial for $\stackrel{\text { bi }}{\otimes}$, but takes some effort to prove in case of $\stackrel{\mathrm{fc}}{\otimes}$. 
As a prerequisite for this proof, in Section 3 we argue that for lattices $A$ and $B$ the tensor product $A \stackrel{\text { fc }}{\otimes} B$ can be represented as a lattice of closed complete bi-ideals in $A \times B$, and that it is a complete meet-subsemilattice of $A \stackrel{\text { bi }}{\otimes}$. Also, we give two characterizations of closed bi-ideals.

In Section 4 we prove the key result of the paper that the tensor product $\stackrel{\text { fc }}{\circledast}$ preserves algebraicity. In fact, we prove that this problem can be reduced to the compactness of propositional logic, see Corollary 1.2.12 in [1]. Thus, we can define finite fc-tensor product of $(0, \vee)$-semilattices, denoted $\underset{\mathrm{fc}}{\mathrm{a}}$, by the formula

$$
A \stackrel{\mathrm{fc}}{\otimes} B=\mathrm{Cp}(\operatorname{Id} A \stackrel{\mathrm{fc}}{\otimes} \operatorname{Id} B) .
$$

Notice that while the tensor products $\stackrel{\text { bi }}{\otimes}$ and $\underset{\otimes}{\otimes}$ are defined in their own right, and $(\dagger)$ simply establishes a relation between them, the alike formula $(\ddagger)$ is used as the definition for $\underset{\mathrm{fc}}{\mathrm{x}}$. Also notice that the "general" tensor product from [4] in our terminology is called finite tensor product, while the tensor product defined in the formal context analysis is the complete fc-tensor product.

Finally, in Section 5 we identify finite fc-tensor product as the lattice tensor product, introduced by G.Grätzer and F.Wehrung in [5]. The key concept on which this definition is based is the box product, thus we will call this tensor product box tensor product. In that paper the authors notice the resemblance of their construction with Wille's construction, in particular that this tensor products coincide in case of finite lattices. Our construction thus can be used to back this resemblance and establish a parallel between the definition of the box tensor product and the finite tensor product.

\section{Motivational EXAMple}

We start with the definition of a tensor product $\stackrel{\text { bi }}{\otimes}$, almost literally repeating the definition of $\underset{\mathrm{bi}}{\bigotimes}$ given in [4].

For a lattice $A$ we call a set $X \subseteq L$ hereditary if $x \in X$ and $y \leqslant x$ implies $y \in X$. For complete lattices $K$ and $L$ we define the complete lateral join as a partial function $\bigvee_{C L}: 2^{K \times L} \rightarrow K \times L$, given by

$$
\bigvee_{C L}\left\langle x_{\alpha}, y_{\alpha}\right\rangle=\left\{\begin{array}{ll}
\left\langle x, \bigvee y_{\alpha}\right\rangle, & \forall \alpha: x_{\alpha}=x ; \\
\left\langle\bigvee x_{\alpha}, y\right\rangle, & \forall \alpha: y_{\alpha}=y
\end{array} .\right.
$$

A subset $I$ of $K \times L$ is called a complete bi-ideal if it is hereditary, it contains the set

$$
\perp_{K, L}=\left(\left\{0_{K}\right\} \times L\right) \cup\left(K \times\left\{0_{L}\right\}\right)
$$

and it is closed under complete lateral joins. We say that $J \subseteq K \times L$ is a complete dual bi-ideal, if $J$ is a bi-ideal in $K^{\mathrm{d}} \times L^{\mathrm{d}}$.

The complete tensor product of $K$ and $L$, denoted $K \otimes$ bi $L$, is the set of complete bi-ideals in $K \times L$ ordered by set inclusion. Obviously, $K \otimes L$ is a complete lattice where the meet coincides with set intersection.

Let $A$ and $B$ be $(0, \vee)$-semilattices, $x \in A$ and $y \in B$. We adopt the conventional notation $(x]$ and $[x)$ for the principal ideal and the principal filter of $x$ in 
$A$ correspondingly. Also, by $(x, y]$ and $[x, y)$ we denote the principal ideal and the principal filter of $(x, y)$ in $A \times B$. The same notation is also used for complete lattices.

We recall that for $(0, \vee)$-semilattices $A$ and $B$, the complete algebraic lattice of all bi-ideals in $A \times B$ is called in [4] an extended tensor product and is denoted $A \bar{\otimes} B$, and $A \bowtie B$ is then defined as the join-semilattice of compact elements of $A \otimes B$.

Lemma 1. For $(0, \vee)$-semilattices $A$ and $B$ the complete lattices $\operatorname{Id} A \stackrel{\text { bi }}{\otimes} \operatorname{Id} B$ and $A \bar{\otimes} B$ are isomorphic, and the isomorphism $\varepsilon: \operatorname{Id} A \otimes$ bi $\operatorname{Id} B \rightarrow A \bar{\otimes} B$ is given by

$$
\varepsilon(\mathcal{I})=\{(x, y) \in A \times B \mid((x],(y]) \in \mathcal{I}\},
$$

for every complete bi-ideal $\mathcal{I}$ in $\operatorname{Id} A \times \operatorname{Id} B$. The inverse mapping takes form

$$
\varepsilon^{-1}(\mathcal{J})=\left\{\left(I_{x}, I_{y}\right) \in \operatorname{Id} A \times \operatorname{Id} B \mid I_{x} \times I_{y} \subseteq \mathcal{J}\right\},
$$

for every bi-ideal $\mathcal{J}$ in $A \times B$.

Proof. We left to the reader the proof of an easy fact that $\varepsilon(\mathcal{I})$ is a bi-ideal and $\varepsilon^{-1}(\mathcal{J})$ is a complete bi-ideal, for any $\mathcal{I} \in \operatorname{Id} A \otimes$ bi $\operatorname{Id} B$ and $\mathcal{J} \in A \bar{\otimes} B$. Let us now prove that $\varepsilon \circ \varepsilon^{-1}$ and $\varepsilon^{-1} \circ \varepsilon$ are identity mappings.

Indeed, for $\mathcal{I} \in \operatorname{Id} A \otimes$ bi $\operatorname{Id} B$ we get

$$
\begin{aligned}
\left(I_{x}, I_{y}\right) & \in \varepsilon^{-1} \circ \varepsilon(\mathcal{I}) \Leftrightarrow I_{x} \times I_{y} \subseteq \varepsilon(\mathcal{I}) \\
& \Leftrightarrow \forall x \in I_{x}, y \in I_{y}:(x, y) \in \varepsilon(\mathcal{I}) \\
& \Leftrightarrow \forall x \in I_{x}, y \in I_{y}:((x],(y]) \in \mathcal{I} \\
& \left.\Leftrightarrow \forall x \in I_{x}: \quad(x], \bigvee_{y \in I_{y}}(y]\right) \in \mathcal{I} \\
& \Leftrightarrow\left(\bigvee_{x \in I_{x}}(x], \bigvee_{y \in I_{y}}(y]\right)=\left(I_{x}, I_{y}\right) \in \mathcal{I} .
\end{aligned}
$$

And for $\mathcal{J} \in A \bar{\otimes} B$ we get

$$
\begin{aligned}
(x, y) & \in \varepsilon \circ \varepsilon^{-1}(\mathcal{J}) \Leftrightarrow((x],(y]) \in \varepsilon^{-1}(\mathcal{J}) \\
& \Leftrightarrow(x] \times(y] \subseteq \mathcal{J} \Leftrightarrow(x, y) \in \mathcal{J} .
\end{aligned}
$$

Let us recall the notions of compactness and algebraicity. An element $x$ in a complete lattice $K$ is compact if $x \leqslant \bigvee S$ for some $S$ implies $x \leqslant \bigvee T$ for some finite $T \subseteq S$. The set $\mathbf{C}(K)$ of all compact elements in a complete lattice $K$ is a $(\vee, 0)$-semilattice of $K$. A complete lattice $A$ is called algebraic if every element is the join of compact elements.

The fact that $A \bar{\otimes} B$ is an algebraic lattice for any $(0, \vee)$-semilattices $A$ and $B$ is thus equivalent to the fact that $\stackrel{\text { bi }}{\otimes}$ preserves algebraicity.

Proposition 1. If $K$ and $L$ are complete algebraic lattices then the lattice $K \otimes L$ is also algebraic. 
Now, using Lemma 1 we get

$$
\begin{aligned}
& A \bowtie B=\operatorname{cp}(\operatorname{Id} A \stackrel{\mathrm{bi}}{\otimes} \operatorname{Id} B), \\
& K \stackrel{\mathrm{bi}}{\otimes} L=\mathrm{Id}(\mathrm{Cp} K \stackrel{\mathrm{bi}}{\otimes} \mathrm{Cp} L),
\end{aligned}
$$

for all $(0, \vee)$-semilattices $A$ and $B$ and all complete algebraic lattices $K$ and $L$.

It is shown in [4] that the bi-ideals can be represented by join-homomorphisms. Below we introduce similar technique for complete bi-ideals.

For complete lattices $K$ and $L$ let us define $K \otimes \vec{\otimes}$ as

$$
K \otimes \vec{\otimes} L=\operatorname{Hom}((K ; \bigvee ; 0),(L ; \bigwedge ; 1)),
$$

that is, $K \otimes \vec{\otimes}$ is a lattice of complete dual join-homomorphisms from $K$ to $L$ sending 0 to 1 .

Proposition 2. For complete lattices $K$ and $L$ the mapping $\eta: K \vec{\otimes} L \rightarrow K \stackrel{\text { bi }}{\otimes} L$ defined by

$$
\eta(\varphi)=\{(x, y) \in K \times L \mid y \leqslant \varphi(x)\},
$$

for any $\varphi \in K \vec{\otimes} L$, establishes an isomorphism between $K \vec{\otimes} L$ and $K \stackrel{\text { bi }}{\otimes} L$. And the inverse mapping is given by

$$
\eta^{-1}(H)(a)=\bigvee\{x \in L \mid(a, x) \in H\},
$$

for any $H \in K \stackrel{\text { bi }}{\otimes} L$ and $a \in L$.

\section{Properties of FC-Tensor product}

The definition of the tensor product in formal concept analysis stems from two papers of R. Wille $[9,10]$. However, in this paper to introduce this tensor product we are following the observational paper of B.Ganter and R.Wille [3]; same results but presented with proofs can be found in sections 4.4 and 5.4 of the monography by the same authors [2].

In formal context analysis the tensor product of complete lattices $K$ and $L$ is defined as the concept lattice

$$
\underline{\mathfrak{B}}(K \times L, K \times L, \nabla),
$$

where $\nabla \subseteq(K \times L) \times(K \times L)$ is a relation defined by

$$
\nabla=\left\{\left(\left(x_{1}, y_{1}\right),\left(x_{2}, y_{2}\right)\right) \mid x_{1} \leqslant x_{2} \text { or } y_{1} \leqslant y_{2}\right\},
$$

see [3]. This concept lattice can be represented as a lattice of subsets of $K \times L$, closed under the closure operator

$$
X \mapsto X^{*+},
$$

where

$$
\begin{aligned}
& X^{*}:=\{b \in K \times L \mid \forall a \in X: a \nabla b\}, \\
& Y^{+}:=\{a \in K \times L \mid \forall b \in Y: a \nabla b\},
\end{aligned}
$$

for all $X, Y \subseteq K \times L$. We call such sets simply closed when the closure operator is clear from the context. Note that the mappings $X \mapsto X^{*}$ and $Y \mapsto Y^{+}$are antitone, 
and thus the mapping $X \mapsto X^{*+}$ is isotone. Also notice that the mapping $X \mapsto$ $X^{+*}$ is also isotone and is a dual closure in $K \times L$. We will take the representation by closed sets as a definition for fc-tensor product, which we denote by $\stackrel{\text { fc }}{\otimes}$. By the properties of the closure operator, the complete meet in $K \otimes$ fc $L$ coincides with set intersection, that is, $K \stackrel{\text { fc }}{\otimes} L$ is a complete meet-subsemilattice in the powerset of $K \times L$.

The following easily verified proposition gives a necessary condition for a set to be closed.

Proposition 3. For complete lattices $K$ and $L$ and $X \subseteq K \times L$, the set $X^{*+}$ is a complete bi-ideal, and $X^{*+}$ is a complete dual bi-ideal.

Thus, $K \stackrel{\mathrm{fc}}{\otimes} L$ is the lattice of closed complete bi-ideals. Further on, we will omit the word "complete" and call them simply closed bi-ideals.

Now we will investigate how the closure operator (1) acts on bi-ideals represented by homomorphisms.

Let $P$ be a poset and $X \subseteq P$. Then we define a hereditary closure of $X$ as a smallest hereditary set containing $X$. One can easily verify that if $Y$ is a hereditary closure of $X$ then it can be represented as

$$
Y=\{y \in P \mid \exists x \in X: y \leqslant x\} .
$$

Lemma 2. For complete lattices $K$ and $L$ and a set $X \subseteq K$ let $Y$ be a hereditary closure of $X$. Then $X^{*+}=Y^{*+}$.

Proof. As $X \subseteq Y$ we get $X^{*+} \subseteq Y^{*+}$. On the other hand, the set $X^{*+}$ is hereditary and contains $X$, thus, it contains its hereditary closure, that is, $Y \subseteq$ $X^{*+}$. But then $Y^{*+} \subseteq X^{*+*+}=X^{*+}$, which proves our claim.

Lemma 3. For complete lattices $K$ and $L$ let $I \subseteq K \times L$ be a set defined as

$$
I=\{(x, y) \mid y \leqslant f(x)\}
$$

for some $f: K \rightarrow L$, and let $f^{*+}=\eta^{-1}\left(I^{*+}\right)$. Then

$$
f^{*+}(x)=\bigwedge_{y \in K-[x)} \bigvee_{w \in K-(y]} f(w)
$$

Note that, in particular, this lemma is applicable in case when $I$ is a complete bi-ideal and $f=\eta^{-1}(I)$

Proof. Let us define the mapping $f^{*}: K \rightarrow L$ as

$$
f^{*}(y)=\bigvee_{w \in K-(y]} f(w),
$$

for all $y \in K$. 
Let $I_{b}=\{(x, f(x)) \mid x \in K\}$, then $I$ is a hereditary closure of $I_{b}$ and by Lemma 2 we get $I^{*}=I_{b}^{*}$. Now

$$
\begin{aligned}
I^{*}=I_{b}^{*} & =\left\{(x, y) \mid \forall x^{\prime}: x^{\prime} \leqslant x \text { or } f\left(x^{\prime}\right) \leqslant y\right\} \\
& =\left\{(x, y) \mid \forall x^{\prime}: x^{\prime} \in K-(x] \text { implies } f\left(x^{\prime}\right) \leqslant y\right\} \\
& =\left\{\begin{array}{c}
\left.(x, y) \mid \bigvee_{x^{\prime} \in K-(x]} f\left(x^{\prime}\right) \leqslant y\right\} \\
\end{array}=\left\{(x, y) \mid f^{*}(x) \leqslant y\right\} .\right.
\end{aligned}
$$

By Proposition 3, the set $I^{*}$ is a complete dual bi-ideal. Taking into account that the mapping $A \mapsto A^{+*}$ is a dual closure, by the same argument as above we get $I^{*+}=I_{c}^{+}$where $I_{c}=\left\{\left(x, f^{*}(x)\right) \mid x \in K\right\}$. Then

$$
\begin{aligned}
I^{*+}=I_{c}^{+} & =\left\{(x, y) \mid \forall x^{\prime}: x \leqslant x^{\prime} \text { or } y \leqslant f\left(x^{\prime}\right)\right\} \\
& =\left\{(x, y) \mid \forall x^{\prime}: x^{\prime} \in K-[x) \text { implies } y \leqslant f^{*}\left(x^{\prime}\right)\right\} \\
& =\left\{(x, y) \mid y \leqslant \bigwedge_{x^{\prime} \in K-[x)} f^{*}\left(x^{\prime}\right)\right\} .
\end{aligned}
$$

This easily yields

$$
f^{*+}(x)=\bigwedge_{x^{\prime} \in K-[x)} f^{*}\left(x^{\prime}\right),
$$

which proves the claim of the theorem.

For complete lattices $K$ and $L$ let us notice that the set $(x, y] \cup \perp$ is a closed bi-ideal, for any $x \in K$ and $y \in L$. Following [4], we call it a pure tensor and denote it by $x \otimes y$. We also introduce a set $[x, y] \subseteq K \times L$ defined by

$$
[x, y]=\left\{\left(x^{\prime}, y^{\prime}\right) \mid x^{\prime} \leqslant x \text { or } y^{\prime} \leqslant y\right\}
$$

Obviously, $[x, y]$ is also a closed bi-ideal, for every $x \in K$ and $y \in L$.

For a set $A \subseteq K \times L$ we define the sets $\left.A\right|_{K} \subseteq K$ and $\left.A\right|_{L} \subseteq L$ as

$$
\begin{aligned}
\left.A\right|_{K} & =\{x \mid \exists y,(x, y) \in A\}, \\
\left.A\right|_{L} & =\{y \mid \exists x,(x, y) \in A\} .
\end{aligned}
$$

We use the symbol $\bigsqcup$ to denote the disjoint union of sets.

Now we give another characterization of the closure operator and, correspondingly, of closed bi-ideals

Lemma 4. For complete lattices $K$ and $L$ and a set $I \subseteq K \times L$ the closure $I^{*+}$ is given by

$$
I^{*+}=\bigcap_{X \sqcup Y=I}\left[\left.\bigvee X\right|_{K},\left.\bigvee Y\right|_{L}\right]
$$




\section{Proof.}

$$
\begin{aligned}
I^{*+} & =\left\{(x, y) \mid \forall\left(x^{\prime}, y^{\prime}\right) \in I^{*}: x \leqslant x^{\prime} \text { or } y \leqslant y^{\prime}\right\} \\
& =\bigcap\left\{\left[x^{\prime}, y^{\prime}\right] \mid\left(x^{\prime}, y^{\prime}\right) \in I^{*}\right\} \\
& =\bigcap\left\{\left[x^{\prime}, y^{\prime}\right] \mid \forall\left(x^{\prime \prime}, y^{\prime \prime}\right) \in I: x^{\prime \prime} \leqslant x^{\prime} \text { or } y^{\prime \prime} \leqslant y^{\prime}\right\} \\
& =\bigcap\left\{\left[x^{\prime}, y^{\prime}\right]|\exists X \subseteq I: \bigvee X|_{K} \leqslant x^{\prime} \text { and }\left.\bigvee(I-X)\right|_{L} \leqslant y^{\prime}\right\} \\
& =\bigcap\left\{\left[\left.\bigvee X\right|_{K},\left.\bigvee Y\right|_{L}\right] \mid X \bigsqcup Y=I\right\} .
\end{aligned}
$$

Corollary 1. The family of closed bi-ideals of $K \times L$ is the minimal family of sets which contains all sets $[x, y]$ and is closed under $\bigcap$.

\section{Algebraicity}

For a $(0, \vee)$-semilattices $A$ and $B$, the extended tensor product $A \bar{\otimes} B$ is an algebraic lattice, see [4]. By Lemma 1 this means that $\stackrel{\text { bi }}{\otimes}$ preserves algebraicity. The goal of this section is to prove similar property for $\stackrel{\text { fc }}{\otimes}$.

Lemma 5. If $K$ and $L$ are complete lattices and $x_{0} \in K$ and $y_{0} \in L$ are compact elements, then $x_{0} \otimes y_{0}$ is a compact element of $K \stackrel{\mathrm{fc}}{\otimes} L$.

Proof. Arguing by contradiction, suppose that $x_{0} \otimes y_{0}$ is not compact. Then there is an infinite family of closed bi-ideals $\left\{I_{\alpha}\right\}_{\alpha \in \mathfrak{A}} \subseteq K \stackrel{\text { fc }}{\otimes} L$ such that

$$
\left(x_{0}, y_{0}\right] \leqslant \bigvee\left\{I_{\alpha} \mid \alpha \in \mathfrak{A}\right\}
$$

and for every finite subfamily $A \subset \mathfrak{A}$ holds

$$
\left(x_{0}, y_{0}\right] \$ \bigvee_{\alpha \in A} I_{\alpha} .
$$

As every bi-ideal from this family can be represented as an infinite join of pure tensors, then, without losing generality, we may assume that every bi-ideal $I_{\alpha}$ is a pure tensor, that is $I_{\alpha}=x_{\alpha} \otimes y_{\alpha}$, for every $\alpha \in \mathfrak{A}$.

As $\bigcup\left\{\left(x_{\beta}, y_{\beta}\right] \mid \beta \in \mathfrak{B}\right\}$ is the hereditary closure of the set $\left\{\left(x_{\beta}, y_{\beta}\right) \mid \beta \in \mathfrak{B}\right\}$, by Lemma 2 we infer

$$
\left(\bigcup\left\{\left(x_{\beta}, y_{\beta}\right] \mid \beta \in \mathfrak{B}\right\}\right)^{*+}=\left(\left\{\left(x_{\beta}, y_{\beta}\right) \mid \beta \in \mathfrak{B}\right\}\right)^{*+},
$$

for every $\mathfrak{B} \subseteq \mathfrak{A}$. Then

$$
\left(x_{0}, y_{0}\right] \leqslant \bigvee_{\alpha \in \mathfrak{A}}\left(x_{\alpha}, y_{\alpha}\right]=\left(\bigcup_{\alpha \in \mathfrak{A}}\left[x_{\alpha}, y_{\alpha}\right)\right)^{*+}=\left\{\left(x_{\alpha}, y_{\alpha}\right) \mid \alpha \in \mathfrak{A}\right\}^{*+},
$$

and using (2) we get

$$
\left(x_{0}, y_{0}\right] \leqslant \bigcap_{\mathfrak{B} \subseteq \mathfrak{A}}\left[\bigvee_{\beta \in \mathfrak{B}} x_{\beta}, \bigvee_{\gamma \in \mathfrak{A}-\mathfrak{B}} y_{\gamma}\right]
$$


And similarly

$$
\left(x_{0}, y_{0}\right] \nless \bigcap_{B \subseteq A}\left[\bigvee_{\beta \in B} x_{\beta}, \bigvee_{\gamma \in A-B} y_{\gamma}\right],
$$

for every finite $A \subseteq \mathfrak{A}$.

Let us define two families $\mathfrak{X}$ and $\mathfrak{Y}$ of finite subsets of $\mathfrak{A}$ by

$$
\begin{aligned}
& \mathfrak{X}=\left\{A \subseteq \mathfrak{A},|A|<\infty \mid x_{0} \leqslant \bigvee_{\alpha \in A} x_{a}\right\}, \\
& \mathfrak{Y}=\left\{A \subseteq \mathfrak{A},|A|<\infty \mid y_{0} \leqslant \bigvee_{\alpha \in A} y_{a}\right\} .
\end{aligned}
$$

That is, $\mathfrak{X}$ and $\mathfrak{Y}$ are the families of all sets of indexes in $\mathfrak{A}$, defining the finite covers of $x_{0}$ and $y_{0}$ correspondingly.

We now need to use some tools from propositional logic, namely the compactness theorem. We are following the terminology of H.J.Keisler and C.C.Chang, see Section 1.2 in [1]. Let us consider the set $\mathfrak{A}$ as the set of simple statements and build a set $\Sigma$ of propositional sentences over it

$$
\begin{aligned}
& \Sigma:=\Sigma_{\mathfrak{X}} \bigsqcup \Sigma_{\mathfrak{Y}}, \\
& \Sigma_{\mathfrak{X}}:=\left\{\neg\left(\bigwedge_{\alpha \in A} \alpha\right) \mid A \in \mathfrak{X}\right\}, \\
& \Sigma_{\mathfrak{Y}}:=\left\{\neg\left(\bigwedge_{\alpha \in A} \neg \alpha\right) \mid A \in \mathfrak{Y}\right\} .
\end{aligned}
$$

Notice that the symbol $\bigwedge$ in the definition above is used not as join, but as a connective in the propositional language. Its usage is justified by the fact that all considered "joins" are finite. The models of our language are simply subsets of $\mathfrak{A}$. For a model $\mathfrak{B} \subseteq \mathfrak{A}$ a simple statement $\beta$ is true in $\mathfrak{B}$ iff $\beta \in \mathfrak{B}$.

We claim that (3) is equivalent to the following statement: The set $\Sigma$ of sentences is not satisfiable, that is, $\Sigma$ has no model. Indeed, (3) can be restated as: For any model $\mathfrak{B} \subseteq \mathfrak{A}$, either $x_{0} \leqslant \bigvee\left\{x_{\beta} \mid \beta \in \mathfrak{B}\right\}$ or $y_{0} \leqslant \bigvee\left\{y_{\gamma} \mid \gamma \in \mathfrak{A}-\mathfrak{B}\right\}$. As $x_{0}$ is compact, it follows that $x_{0} \leqslant \bigvee\left\{x_{\beta} \mid \beta \in \mathfrak{B}\right\}$ iff there is $A_{0} \in \mathfrak{X}$ such that $A_{0} \subseteq \mathfrak{B}$, in which case the propositional sentence

$$
\left(\neg \bigwedge\left\{a \mid a \in A_{0}\right\}\right) \in \Sigma_{\mathfrak{X}}
$$

is not satisfied in $\mathfrak{B}$. Similarly, $y_{0} \leqslant \bigvee\left\{y_{\gamma} \mid \gamma \in \mathfrak{A}-\mathfrak{B}\right\}$ iff there is a set $B_{0} \in \mathfrak{Y}$ such that $B_{0} \subseteq \mathfrak{A}-\mathfrak{B}$, and consequently the propositional sentence

$$
\left(\neg \bigwedge\left\{\neg a \mid a \in B_{0}\right\}\right) \in \Sigma_{\mathfrak{Y}}
$$

is not satisfied in $\mathfrak{B}$. Combined together, these observations prove our claim.

Similarly, (4) is equivalent to the statement: The set $\Sigma$ of sentences is finitely satisfiable, that is, every finite subset of $\Sigma$ has a model. However, by the compactness theorem for propositional calculus, see Corollary 1.2.12 in [1], $\Sigma$ is satisfiable if it is finitely satisfiable, a contradiction.

Now as an easy corollary we get

Theorem 1. If $K$ and $L$ are complete algebraic lattices then $K^{\mathrm{fc}} L^{\circ}$ is algebraic. 
Proof. Notice that by Lemma $5, x \otimes y$ is a compact element of $K \stackrel{\mathrm{fc}}{\otimes} L$, for any $x \in \mathrm{Cp} K$ and $y \in \mathrm{Cp} L$. Any closed bi-ideal $I \subseteq K \times L$ can be represented as

$$
\begin{aligned}
I & =\perp \cup \bigcup\{(x, y] \mid(x, y) \in I, x \in \mathrm{Cp} K, y \in \mathrm{Cp} L\} \\
& =\bigvee\{x \otimes y \mid(x, y) \in I, x \in \operatorname{Cp} K, y \in \operatorname{Cp} L\} .
\end{aligned}
$$

Thus, every element of $K \stackrel{\mathrm{fc}}{\otimes} L$ can be represented as a join of compact elements, so $K \stackrel{\text { fc }}{\otimes} L$ is algebraic.

Now, using Theorem 1 , for $(0, \vee)$-semilattices $A$ and $B$ we can define finite $f c$ tensor product as

$$
A \stackrel{\mathrm{fc}}{\otimes} B=\mathrm{Cp}(\operatorname{Id} A \stackrel{\mathrm{fc}}{\otimes} \operatorname{Id} B) .
$$

and observe that, just as for "regular" tensor product, holds

$$
K \stackrel{\mathrm{fc}}{\otimes} L=\operatorname{Id}(\operatorname{Cp} K \stackrel{\mathrm{fc}}{\otimes} \operatorname{Cp} L),
$$

for all complete algebraic lattices $K$ and $L$.

\section{The BOX Tensor PROduCt}

Now we are going to show that, thus defined, finite fc-tensor product coincides with the box tensor product, introduced in [5].

For lattices with zero $A$ and $B, a \in A$ and $b \in B$ we define the box tensor product of $A$ and $B$, denoted $A \bowtie B$, as the set of all finite intersections of the form

$$
H=\bigcap\left\{\left[a_{i}, b_{i}\right] \mid i<n\right\}
$$

satisfying

$$
\begin{aligned}
& \bigwedge\left\{a_{i} \mid i<n\right\}=0_{A}, \\
& \bigwedge\left\{b_{i} \mid i<n\right\}=0_{B},
\end{aligned}
$$

where $n>0,\left(a_{i}, b_{i}\right) \in A \times B$, for all $i<n$.

Let us point out that in making this definition we have skipped few intermediate steps as compared to [5]; the definition now corresponds to Lemma 3.8 of the mentioned paper.

Now, let us identify $A$ and $B$ with the sets of principal ideals in $\operatorname{Id} A$ and $\operatorname{Id} B$ correspondingly, using canonical embeddings $\pi_{A}: x \mapsto(x]$ and $\pi_{B}: y \mapsto(y]$; and let us extend this embeddings to the embedding $\pi: A \bowtie B \rightarrow A \stackrel{\text { fc }}{\otimes} B$ defined by

$$
\pi: \bigcap\left\{\left[a_{i}, b_{i}\right] \mid i<n\right\} \mapsto \bigcap\left\{\left[\pi_{A}\left(a_{i}\right), \pi_{B}\left(b_{i}\right)\right] \mid i<n\right\} .
$$

Notice that by Corollary 1 , all elements of $\pi[H]$ are valid elements of $\operatorname{Id} A \stackrel{\text { fc }}{\otimes} \operatorname{Id} B$, that is, valid closed bi-ideals in $\operatorname{Id} A \times \operatorname{Id} B$.

For a lattice $C$ and a set $X \subseteq C$ we denote the lattice generated by $X$ in $C$ by $\langle X\rangle_{C}$, or simply by $\langle X\rangle$ if the underlying lattice is clear from the context. 
Proposition 4. For lattices with zero $C$ and $D$, let $c_{i} \in C$ and $d_{i} \in D$, for $i=1, \ldots, n$. Then

$$
\bigcap_{i=1, \ldots, n}\left[c_{i}, d_{i}\right]=\perp_{c_{i}, d_{i}} \cup \bigcup_{i=1, \ldots, m}\left(z_{i}, w_{i}\right]
$$

where

$$
\perp_{c_{i}, d_{i}}=\left(\left(\bigwedge c_{i}\right] \times D\right) \cup\left(C \times\left(\bigwedge d_{i}\right]\right)
$$

for some $m$, and $z_{i} \in\left\langle c_{j} \mid j=1, \ldots, n\right\rangle$ and $w_{i} \in\left\langle d_{j} \mid j=1, \ldots, n\right\rangle$, for all $i$.

Using Proposition 4 we can easily prove the desired result.

Theorem 2. For lattices with zero $A$ and $B$, the mapping $\pi$ defined by (8) establishes an isomorphism between $A \bowtie B$ and $A$ 兴 $B$, that is

(1) for any $H \in A \otimes B, \pi(H)$ is a compact closed bi-ideal in $\operatorname{Id} A \times \operatorname{Id} B$,

(2) any compact compact closed bi-ideal in $\operatorname{Id} A \times \operatorname{Id} B$ can be represented as $\pi(H)$, for some $H \in A \bowtie B$.

Proof. (1): Let us take $H$ as in (5), then

$$
\pi(H)=\bigcap\left\{\left[\pi_{A}\left(a_{i}\right), \pi_{B}\left(b_{i}\right)\right] \mid i<n\right\},
$$

and, by Proposition 4

$$
\pi(H)=\perp_{\pi_{A}\left(a_{i}\right), \pi_{B}\left(b_{i}\right)} \cup \bigcup_{i=1, \ldots, m}\left(z_{i}, w_{i}\right],
$$

where $z_{i} \in\left\langle\pi_{A}\left(a_{j}\right) \mid j=1, \ldots, n\right\rangle \subseteq \pi_{A}[A]$ and $w_{i} \in\left\langle\pi_{B}\left(b_{j}\right) \mid i=1, \ldots, n\right\rangle \subseteq$ $\pi_{B}[B]$.

Also from (6) and (7) it follows that $\perp_{\pi_{A}\left(a_{i}\right), \pi_{B}\left(b_{i}\right)}=\perp_{\operatorname{Id} A \text {,Id } B}$, which yields

$$
\pi(H)=\bigcup_{i=1, \ldots, m} z_{i} \otimes w_{i}=\bigvee_{i=1, \ldots, m} z_{i} \otimes w_{i},
$$

where $z_{i}$ and $w_{i}$ are compact elements of $\operatorname{Id} A$ and $\operatorname{Id} B$ correspondingly, for all $i \leqslant n$. Thus, by Lemma 5 the elements $z_{i} \otimes w_{i}$ are compact elements of $\operatorname{Id} A \stackrel{\mathrm{fc}}{\otimes} \operatorname{Id} B$ and $\pi(H)$ is a finite join of compact elements, thus, it is also compact.

(2): Let $\mathcal{H}$ be a closed compact bi-ideal in $\operatorname{Id} A \times \operatorname{Id} B$. Definitely, $\mathcal{H}$ can be represented as

$$
\begin{aligned}
\mathcal{H} & =\bigcup\left\{a \otimes b \mid a \in \pi_{A}[A], b \in \pi_{B}[B],\left(\pi_{A}(a), \pi_{B}(b)\right) \in H\right\} \\
& =\bigvee\left\{a \otimes b \mid a \in \pi_{A}[A], b \in \pi_{B}[B],\left(\pi_{A}(a), \pi_{B}(b)\right) \in H\right\} .
\end{aligned}
$$

Using compactness of $\mathcal{H}$, we get

$$
\mathcal{H}=\bigvee\left\{a_{i} \otimes b_{i} \mid i<n\right\}=\left(\bigcup\left\{a_{i} \otimes b_{i} \mid i<n\right\}\right)^{*+},
$$

for some $n>0$ and $a_{i} \in \pi_{A}[A]$ and $b_{i} \in \pi_{B}[B]$,for all $i<n$. By Lemma 2 the latter gives

$$
\mathcal{H}=\left(\bigcup\left\{\left(a_{i}, b_{i}\right) \mid i<n\right\}\right)^{*+},
$$

and the application of Lemma 4 gives us the desired representation of $\mathcal{H}$. 


\section{Conclusion}

The paper [5] contains a very profound discussion on the similarities between various kinds of tensor products and their properties, as well as a list of open problems. In this list we would like to single out two problems that explicitly deal with the connection between the tensor box product and finite tensor product. Problem 1 asks for a characterization of a situation when $A \bowtie B=A \bowtie B$, for lattices with zero $A$ and $B$, and Problem 6 seeks for another tensor products "between" $\square$ and $\mathrm{bi}$.

A problem in comparing these tensor products is that the finite tensor product is defined on $(0, \vee)$-semilattices, while the box tensor product is defined on lattices with zero. This situation is natural in the following sense: for lattices with zero $A$ and $B, A \bowtie B$ is always a lattice, while $A \bowtie B$ in general is only a $(0, \vee)$-semilattice. Now, our construction of $₫$ enables to extend the definition of the box tensor product to $(0, \vee)$-semilattices, and thus enables to compare these constructions on some "natural" domain. Again, correspondences $(\dagger)$ and $(\ddagger)$ enable to characterize

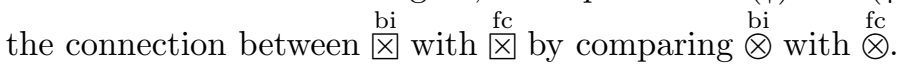

The author would also like to draw the parallel to the paper of M. Krötzsch and G. Malik [8]. For complete lattices $K$ and $L$, the space of regular Galois connections described in this paper is $F \stackrel{\text { fc }}{\otimes} L$; indeed, regular Galois connections are deliberately defined this way. In the same time one can show, not without some effort, that the space of all Galois connections will be exactly $F \stackrel{\text { bi }}{\otimes} L$. The large part of [8] is dedicated to describing the situation when $K$ and $L$ have only regular Galois connections between them, and in particular to the case when $K$ have only regular Galois connections to any complete lattice $L$.

Regarding the latter case, the author believe that he has the proof that this holds iff $K$ satisfies complete infinite distributive identity (CIDI). The one direction of this statement is provided by Theorem 4 of [8]. For the other direction, when $K$ does not satisfy CIDI, the counterexample is provided by the identity mapping from $K$ to $K^{\mathrm{op}}$, which would be an irregular Galois connection. However, the complete proof of this fact requires efforts which fall beyond the scope of the present article.

\section{REFERENCES}

[1] C.C.Chang, H.J.Keisler, Model Theory, 3d ed., North-Holland, Amsterdam, 1990.

[2] B.Ganter, R.Wille, Formal concept analysis - mathematical foundations, Springer-Verlag, 1999.

[3] B.Ganter, R.Wille, Applied lattice theory: formal concept analysis, Appendix H in [7], 591-605.

[4] G.Grätzer, F.Wehrung, A survey of tensor products and related constructions in two lectures, Algebra Universalis 45 (2001), 117-134.

[5] G.Grätzer, F.Wehrung, A new lattice construction: the box product, J. Algebra 221 (1999), 315-344.

[6] G.Grätzer, F.Wehrung, Tensor products and transferability of semilattices, Canad. J. Math. 51 (1999), 792-815.

[7] G.Grätzer, General Lattice Theory, 2nd ed., Birkhäuser-Verlag, Basel, 1998.

[8] M. Krötzsch, G.Malik, The Tensor Product as a Lattice of Regular Galois Connections, ICFCA (2006), 89-104.

[9] R.Wille, Tensorial decompositions of concept lattices, Order 2 (1985), 81-95. 
[10] R.Wille, Tensor products of complete lattices as closure systems, Contributions to General Algebra 7 (1991), 381-386.

Department of Computer Science, V.N. Karazin Kharkiv National University, Kharkiv, UKRAINE

E-mail address, B. Chornomaz: markyz.karabas@gmail.com 